\title{
Modelling Activity and Durability of Electrocatalysts from a Statistical Perspective
}

\author{
Jun Huang \\ Institute of Theoretical Chemistry, Ulm University, 89069 Ulm Germany \\ Email address: jun.huang@uni-ulm.de
}

\begin{abstract}
We develop a theory to investigate how energetic nonhomogeneity of active sites determines the overall activity of an electrocatalyst and how the evolution of the nonhomogeneity determines the overall durability. The simple theory is amenable to exact analytical solutions and thus fosters an in-depth transparent analysis. It is revealed that nonhomogeneity does not necessarily diminish the electrocatalytic activity; instead, the highest overall activity is obtained with a suitable level of nonhomogeneity that is commensurate with the mean property. The evolution kinetics of nonhomogeneity is described by using the Fokker-Planck theory. Exponential decay of the activity is predicted theoretically and confirmed experimentally. The present work represents a first step toward closing the gap between model and practical electrocatalysts using statistical considerations.
\end{abstract}

\section{Main text}

Electrocatalysts can alter the reaction path and lower the activation barrier of a reaction while retaining their own identity and integrity.[1] In many applications, such as fuel cells and water splitting devices, electrocatalysts are usually present in the form of nanoparticles because of their increased surface area. In contrast to model electrocatalysts, e. g., atomically well-defined single crystals, [2-4] real-world electrocatalysts have a large variety of active sites on the atomic level.[5,6] These active sites reveal a dissimilar electrocatalytic activity as demonstrated in high-resolution catalytic activity mapping studies.[7-12] Consequently, it is indispensably required to understand the influence of nonhomogeneously distributed active sites since this knowledge is important to the rational design of electrocatalysts.[13]

An electrocatalytic reaction, for example, the oxygen reduction reaction (ORR), is a reaction network consisting of several elementary steps and different adsorbing reaction intermediates. Interlinking the electrocatalytic activity with binding energies of adsorbing intermediates is a major straddle in comprehending and controlling the structure dependence of electrocatalysis.[14-17] Furthermore, binding energies of different intermediates are linearly correlated with each other among a wide range of electrocatalysts.[18,19] Consequently, one of the binding energies can be chosen as the descriptor of a class of materials. The relation between the electrocatalytic activity and the 
chosen descriptor usually manifests in a volcano plot; that is, the activity increases until a maximum is reached, and then declines as the descriptor is varied from small to large values. The basic idea refers to the well-known Sabatier principle[20], indicating that the optimum electrocatalyst binds the reaction intermediates neither too strongly nor too weakly.[1,21] This binding-energy approach prevails, with proven efficacy, in electrocatalysis, and the vast majority of research aspires to climb up the volcano apex.[22,23]

In line with the binding-energy approach, it appears viable to endow the chosen descriptor with a probability distribution function (PDF) for different active sites. This will contribute to answer the question how the active-site distribution influences the overall activity and durability of an electrocatalyst. So far, only a few works have addressed such issues. Norskov et al. presented a conceptual analysis on the varying contributions of different types of active sites to the overall activity.[1] Eikerling et al. systematically studied the degradation of the electrochemical surface area of Pt-based ORR catalysts.[24-26] In this Letter, we develop a new statistical theory for understanding the activity and durability of electrocatalysts from the perspective of spatially distributed and temporally evolving binding energy at active sites. Our theory is universally applicable for heterogeneous electrocatalysis and may spur further progress in a rational design of active sites.

\section{Theory}

We consider a nonhomogeneous electrocatalyst, as shown schematically in Figure 1 (a), with nonhomogeneously distributed active sites. The nonhomogeneity results in a bindingenergy distribution characterized by a probability density function (PDF). The phenomenon that the activity of the electrocatalyst changes as a function of time is described as a consequence of the evolution of the PDF. The task is to interlink the overall activity with parameters to the PDF, and further to connect the degradation of the electrocatalytic activity to the evolution kinetics of the PDF.

We assume the activity descriptor's PDF as a normal distribution, depicted in Figure 1 (c) and given by

$$
p(x)=\frac{1}{\sqrt{2 \pi} \sigma} \exp \left(-\frac{1}{2}\left(\frac{x-\mu}{\sigma}\right)^{2}\right),
$$

where $x(\mathrm{eV})$ is the activity descriptor, $\mu(\mathrm{eV})$ the mean value, and $\sigma(\mathrm{eV})$ the standard deviation. We assume that the electrocatalytic activity is a symmetric volcano-shaped function of the descriptor, Figure 1 (b). After normalization with respect to the maximum activity, the volcano plot is expressed as

$$
f(x)=\exp (-\beta|x|),
$$

where the coefficient is $\beta=\left(2 k_{B} T\right)^{-1}$ with $k_{B}\left(\mathrm{eV} \mathrm{K}^{-1}\right)$ being the Boltzmann's constant and $T(\mathrm{~K})$ the absolute temperature. At $T=300 \mathrm{~K}$, we have $\beta=19.4 \mathrm{eV}^{-1}$. As volcano plots are potential-dependent,[27-30] it is thus important to note that the present theory describes the performance of electrocatalysts at constant potential, rather than constant current (density). The specific forms of $p(x)$ and $f(x)$ are reasonable in terms of physical 
significance, and also simple in terms of algebraic manipulation. The subsequent derivation is generic to other forms of $p(x)$ and $f(x)$, for example, asymmetric volcano-shaped functions.

(a)

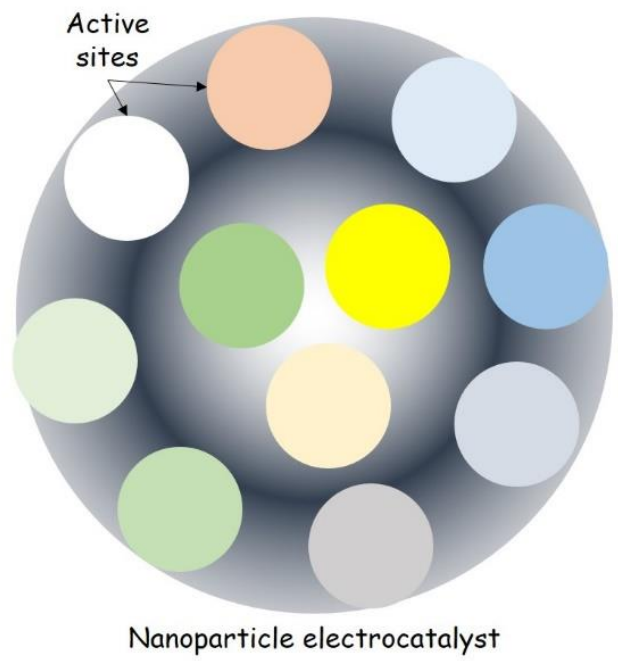

(b)

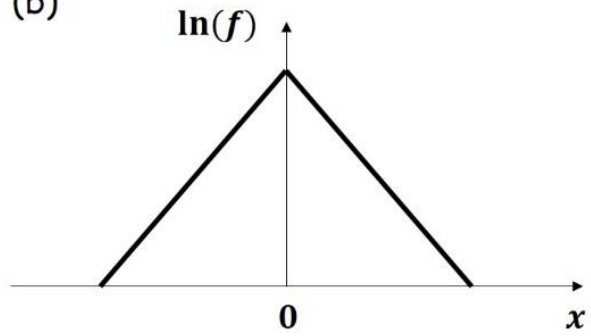

(c)

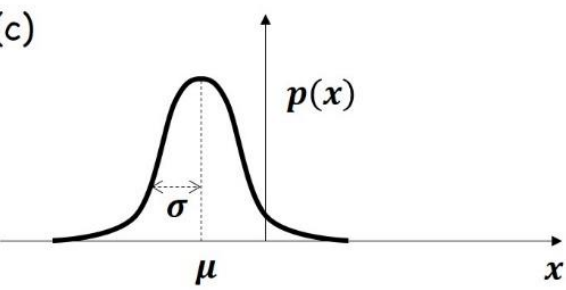

Figure 1. (a) Schematic illustration of a nonhomogeneous electrocatalytic particle. Circles in different colors represent active sites with different binding energies. (b) shows the volcano-shaped activity function $f(x)=$ $\exp (-\beta|x|)$ with $\beta$ being the exponential coefficient. The vertical axis has a logarithmic scale. (c) shows a normal distribution of binding energy, viz., the activity descriptor, among different active sites $p(x)$ with a mean value of $\mu(\mathrm{eV})$ and a standard deviation of $\sigma(\mathrm{eV})$.

The fraction of active sites falling into the descriptor range of $[x, x+d x]$ is $p(x) d x$, each contributing to the overall activity with an amount of $f(x)$. The overall activity is the sum of all active sites

$$
\langle f\rangle=\int_{-\infty}^{\infty} d x f(x) p(x) .
$$

Some algebraic manipulations result in an analytical solution for $\langle f\rangle$

$$
\begin{aligned}
\langle f\rangle=\frac{1}{2}(\exp ( & \left.\frac{\beta^{2} \sigma^{2}+2 \beta \mu}{2}\right) \operatorname{erfc}\left(\frac{\beta \sigma^{2}+\mu}{\sqrt{2} \sigma}\right) \\
& \left.+\exp \left(\frac{\beta^{2} \sigma^{2}-2 \beta \mu}{2}\right) \operatorname{erfc}\left(\frac{\beta \sigma^{2}-\mu}{\sqrt{2} \sigma}\right)\right),
\end{aligned}
$$

where $\operatorname{erfc}(\mathrm{x})$ is the complementary error function.

Figure 2 (a) depicts the variation of $\langle f\rangle$ as a function of $\sigma$ for different values of $\mu$. Since $\langle f\rangle$ is an even function of $\mu$, we only have to consider positive values of $\mu$, corresponding to the weak-binding leg of the volcano plot. The analysis applies equally to the strongbinding leg of the volcano, i. e., $\mu<0$. When $\mu=0$ (the mean binding energy is reconciled with the volcano's apex), $\langle f\rangle$ monotonically decreases as $\sigma$ grows, implying 
that the overall activity of an averagely optimal electrocatalyst decreases rapidly when the homogeneity deteriorates. When $\mu \neq 0,\langle f\rangle$ changes nonmonotonically with increasing $\sigma$, implying that nonhomogeneity is, surprisingly, not always detrimental. Specifically, $\langle f\rangle$ increases when $\sigma$ reaches $\sim \mu$, and then decreases slowly. An intuitive explanation of this peculiar nonmonotonicity is that a certain degree of nonhomogeneity, commensurate with the mean property, renders the highest fraction of active sites near the volcano's apex. In other words, for the vast majority of electrocatalysts whose mean property deviates from the optimum, the overall activity is highest when $\sigma \approx \mu$ instead of the expected outcome $\sigma=0$.
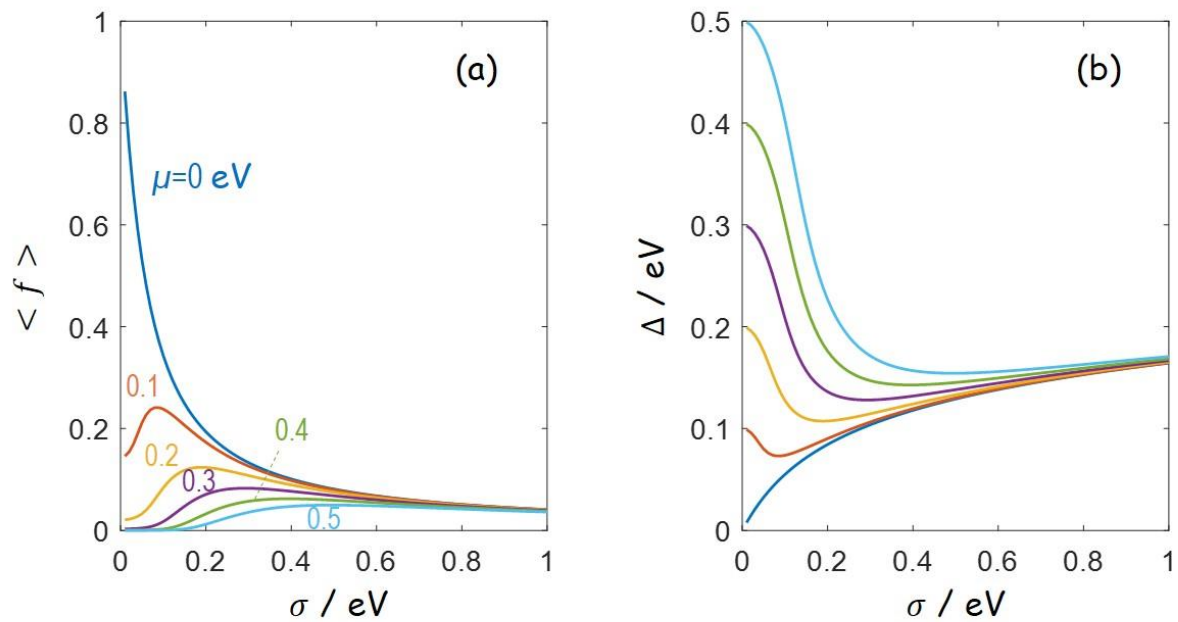

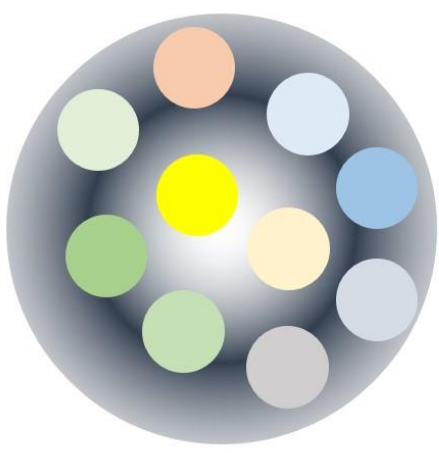

Nonhomogeneous

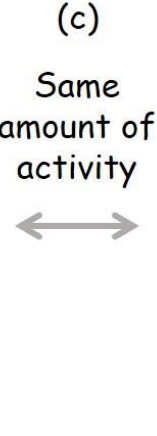

Same amount of activity

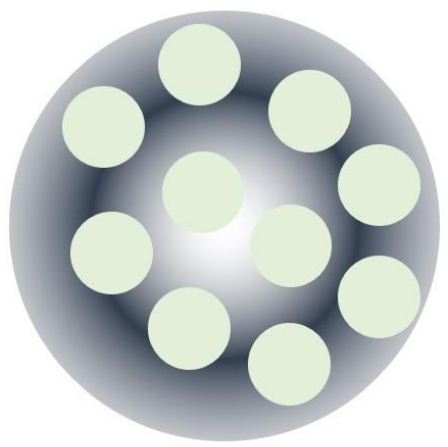

Homogeneous

Figure 2. (a) The overall activity $\langle f\rangle$ and (b) the positive-defined descriptor value of the homogenous counterpart $\Delta$ as a function of $\sigma$ for different values of $\mu$ (in steps of $0.1 \mathrm{eV}$ from 0 to $0.5 \mathrm{eV}$ ). (c) Schematic illustration of the homogeneous counterpart of a nonhomogeneous electrocatalyst.

\section{Homogeneous counterpart}

A homogeneous counterpart is a homogeneous electrocatalyst $(\sigma=0)$ that provides the same amount of activity as that of the nonhomogeneous electrocatalyst, c.f. Figure 2(c). The descriptor value of this homogenous counterpart is denoted as $\Delta(\mathrm{eV})$ and adopts positive values only (note again that $\langle f\rangle$ is an even function of $x$ ). From the definition, we have $f(\Delta)=\langle f\rangle$, leading to 


$$
\Delta(\mu, \sigma)=-\frac{\beta \sigma^{2}}{2}-\frac{1}{\beta} \ln \left(\frac{e^{\beta \mu} \operatorname{erfc}\left(\frac{\beta \sigma^{2}+\mu}{\sqrt{2} \sigma}\right)+e^{-\beta \mu} \operatorname{erfc}\left(\frac{\beta \sigma^{2}-\mu}{\sqrt{2} \sigma}\right)}{2}\right) .
$$

When $\mu \neq 0, e^{\beta|\mu|} \operatorname{erfc}\left(\frac{|\mu|+\beta \sigma^{2}}{\sqrt{2} \sigma}\right) \ll e^{-\beta|\mu|} \operatorname{erfc}\left(\frac{\beta \sigma^{2}-|\mu|}{\sqrt{2} \sigma}\right)$, and $\Delta$ is approximated as

$$
\Delta(\mu \neq 0) \approx|\mu|-\frac{\beta \sigma^{2}}{2}-\frac{1}{\beta} \ln \left(\frac{1}{2} \operatorname{erfc}\left(\frac{\beta \sigma^{2}-|\mu|}{\sqrt{2} \sigma}\right)\right) .
$$

When $\mu=0$, Eq.(5) is reduced to

$$
\Delta(\mu=0)=\frac{\beta \sigma^{2}}{2}-\frac{1}{\beta} \ln \left(\operatorname{erfc}\left(\frac{\beta \sigma}{\sqrt{2}}\right)\right)
$$

which, by using the approximation, $\operatorname{erfc}(\mathrm{x}) \approx \frac{1}{\sqrt{\pi}} \frac{e^{-x^{2}}}{x}$ for $x \gg \sqrt{2 / \pi}$, namely, $\sigma \gg$ $\frac{2}{\beta} \sqrt{\frac{2}{\pi}}=0.08 \mathrm{eV}$, could be further simplified to

$$
\Delta\left(\mu=0, \sigma \gg \frac{2}{\beta} \sqrt{\frac{2}{\pi}}\right)=\frac{1}{\beta} \ln \left(\sqrt{\frac{\pi}{2}} \beta \sigma\right) .
$$

Figure 2(b) visualizes how $\Delta$ varies as a function of $\mu$ and $\sigma$. At the vertical line $\sigma=0$, namely, for homogeneous electrocatalysts, we retrieve the expected result, $\Delta=\mu$. For a given $\mu, \Delta$ first decreases but then increases with raising $\sigma$, which is consistent with the nonmonotonic trend of $\langle f\rangle$ in Figure 2(a). All the curves converge to the same curve, $\Delta=$ $\frac{1}{\beta} \ln \left(\sqrt{\frac{\pi}{2}} \beta \sigma\right)$, when $\sigma \gg \max \left(\frac{2}{\beta} \sqrt{\frac{2}{\pi}}, \sqrt{\frac{|\mu|}{\beta}}\right)$, implying that the overall activity is nearly independent of the mean property when the standard deviation is sufficiently high. In other words, homogeneity control becomes more important than mean-property control in such situations.

\section{Site-site variation}

In addition to the overall activity, we are also interested in the activity variation between different active sites, termed site-site variation for short. Therefore, it is desirable to interlink the site-site variation with the PDF. An analytical expression for the site-site variation normalized with respect to the average activity $\Sigma$, is obtained 


$$
\Sigma=\sqrt{\frac{2 e^{(\beta \sigma)^{2}}\left[e^{-2 \beta \mu} \operatorname{erfc}\left(\frac{2 \beta \sigma^{2}-\mu}{\sqrt{2} \sigma}\right)+e^{2 \beta \mu} \operatorname{erfc}\left(\frac{2 \beta \sigma^{2}+\mu}{\sqrt{2} \sigma}\right)\right]}{\left[e^{-\beta \mu} \operatorname{erfc}\left(\frac{\beta \sigma^{2}-\mu}{\sqrt{2} \sigma}\right)+e^{\beta \mu} \operatorname{erfc}\left(\frac{\beta \sigma^{2}+\mu}{\sqrt{2} \sigma}\right)\right]^{2}}}-1
$$

Figure 3 shows $\Sigma$ as a function of $\sigma$ when $\mu$ varies from $0 \mathrm{eV}$ to $1 \mathrm{eV}$ (in steps of $0.1 \mathrm{eV}$ ). $\Sigma$ first increases exponentially, but then goes through a hump, and finally converges, regardless of $\mu$, to $\sigma^{0.5}$ with increasing values of $\sigma$. For a given $\sigma, \Sigma$ is greater with increasing $\mu$. Correspondingly, the activity variation between active sites is more pronounced when the mean binding energy deviates further from the volcano's apex. The nonmonotonic behavior of $\Sigma$ as a function of $\sigma$ for $\mu>0 \mathrm{eV}$ can be understood in the same way as in Figure 2 (a) and (b) corresponding to $\langle f\rangle$ and $\Delta$, respectively.

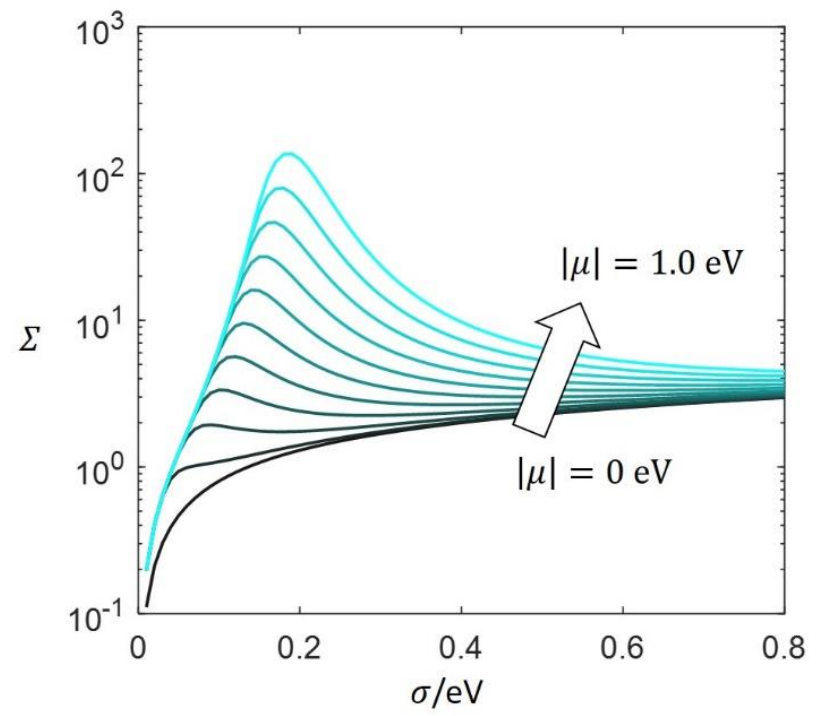

Figure 3. Normalized site-site variation $\Sigma$ as a function of $\sigma$ when $|\mu|$ increases from $0 \mathrm{eV}$ to $1 \mathrm{eV}$ in steps of $0.1 \mathrm{eV}$.

\section{Degradation of activity}

After examining the electrocatalyst at a certain point of its lifetime, we proceed to study the degradation behavior. An electrocatalyst may degrade due to a plethora of mechanisms, such as, active site poisoning, support corrosion, dissolution and agglomeration of active sites, etc.[31-33] A comprehensive treatment of the degradation is beyond the scope of this work. Instead, we focus on interlinking the degradation of the activity with the evolution of the binding energy of active sites, which can be attributed to atomic mechanisms such as active-site poisoning, change in electronic and geometrical factors of the electrocatalyst, and change in the local reaction condition near active sites.[31-34] Consequently, the task of modelling the degradation of the activity is transformed to that of modelling the evolution of the descriptor's PDF, $p(x)$. 
Treated as a Markovian stochastic process, the PDF evolution process is described using the Fokker-Planck theory [35]

$$
\frac{\partial p(x, t)}{\partial t}=\frac{\partial}{\partial x}\left(-\gamma(x, t) p(x, t)+D(x, t) \frac{\partial p(x, t)}{\partial x}\right)
$$

where the first term on the right hand side is a drift flux, and the second term a diffusion flux. Generally, the drift coefficient $\gamma(x, t)$ and the diffusion coefficient $D(x, t)$ are timevarying and dependent on $x$. The usual case where the volcano peak repels active sites away from the volcano peak $(x=0)$ and the overall activity decreases during aging is described by $\gamma(x, t)>0$.

We assume that the initial distribution is a normal distribution

$$
p(x, 0)=\frac{1}{\sqrt{2 \pi} \sigma_{0}} \exp \left(-\frac{\left(x-\mu_{0}\right)^{2}}{2 \sigma_{0}^{2}}\right),
$$

with $\mu_{0}$ and $\sigma_{0}$ being the initial mean value and standard deviation. A zero probability at infinity serves as the other boundary condition closing Eq.(10)

$$
p( \pm \infty, t)=0
$$

Assuming constant $\gamma$ and $D$, we obtain an analytical expression of $p(x, t)$

$$
p(x, t)=\frac{1}{\sqrt{2 \pi} \sigma(t)} \exp \left(-\frac{(x-\mu(t))^{2}}{2 \sigma(t)^{2}}\right)
$$

with a time-varying mean value

$$
\mu(t)=\mu_{0} \exp (\gamma t)
$$

and a time-varying standard deviation

$$
\sigma(t)=\sqrt{\frac{D(\exp (2 \gamma t)-1)}{\gamma}+\sigma_{0}^{2} \exp (2 \gamma t)} .
$$

The PDF's evolution is characterized by two phenomenological parameters $\gamma$ and $D$. Specifically, the growth rate of $\mu$ is solely determined by $\gamma$, while that of $\sigma$ is codetermined by $\gamma$ and $D$, and $\sigma(t)=\sqrt{2 D t+\sigma_{0}^{2}}$ if $\gamma=0$. Figure 4 (a)-(c) shows the evolution of $p(x, t)$ for different initial conditions with a diffusion coefficient of $D=$ $0.005\left(\mathrm{~m} \mathrm{eV}^{-2} \mathrm{~s}^{-1}\right.$ ) (An arbitrary value for the mere purpose of quantitative exposition; the results of Figure 4 (a)-(c) do not change if we use other values). For $\mu_{0}<0\left(\mu_{0}>0\right)$, the mean of $p(x, t)$ is shifted to more negative (positive) values and $\sigma$ increases with aging. In case of $\mu_{0}=0$, however, the mean of $p(x, t)$ is kept virtually constant while $\sigma$ grows. 
As $p(x, t)$ retains a normal distribution along aging, the overall activity $\langle f\rangle$ is still expressed as Eq.(13). The degradation pattern, namely, the time dependency of $\langle f\rangle$, is dependent on the initial distribution. If $\left|\mu_{0}\right|>\beta \sigma_{0}^{2}$, the degradation behavior initially follows, $\ln \langle f\rangle \propto-\beta\left[\gamma\left(\left|\mu_{0}\right|-\beta \sigma_{0}^{2}\right)+D\right] t$ and transitions to, $\ln \langle f\rangle \propto-\gamma t$, at a later stage. If $\left|\mu_{0}\right|<\beta \sigma_{0}^{2}$, only the latter pattern, $\ln \langle f\rangle \propto-\gamma t$, can be found. Figure 4 (d)-(e) shows the evolution of $\langle f\rangle$, normalized to its initial value, for different $\mu_{0}$ 's and different $D$ 's, respectively. Given $\sigma_{0}=0.05 \mathrm{eV}$ and $D=\gamma \sigma_{0}^{2} / 2, \ln \langle f\rangle$ decreases with a slope of -1 as a function of $\gamma t$ for $\mu_{0}=0 \mathrm{eV}$, while it exhibits a two-stage degradation pattern for $\mu_{0}=$ $-0.2 \mathrm{eV}$, viz., $\left|\mu_{0}\right|>\beta \sigma_{0}^{2}$. The first stage becomes steeper when $D$ is greater, as shown in Figure 4 (e).

The theoretical prediction of exponential decay in activity is tested using platinum-group metal-free (PGM-free) ORR electrocatalysts. This is done for two different reasons. Firstly, the present theory describes electrocatalytic activity and durability at a fixed potential. Therefore, it is a requirement of consistency that experimental data used to test the theory should also be obtained at a fixed potential, which is the usual case for PGM-free ORR electrocatalysts. On the contrary, the durability of Pt-based ORR electrocatalysts is evaluated, according to standard protocols,[36] by letting electrocatalysts undergo potential cycling. Secondly, the present theory describes activity degradation due to evolution of the binding-energy distribution, in accordance with the major atomic degradation mechanisms of PGM-free ORR electrocatalysts, namely, active site poisoning and deactivation.[31-33] As for Pt-based ORR electrocatalysts, the major degradation mechanisms are Pt dissolution, agglomeration, and detachment.[24-26]

Figure 4 (f)-(g) show the activity degradation of two PGM-free ORR electrocatalysts belonging to the Fe/N/C catalyst family, including (f) the type A prepared by Shao et al.[34], and (g) the type B prepared by Yin and Zelenay.[37] Three type A electrocatalysts, prepared at three pyrolysis temperatures $\left(950,1050,1150{ }^{\circ} \mathrm{C}\right)$, are compared at $0.6 \mathrm{~V}_{\mathrm{RHE}}$ in a single $\mathrm{H}_{2} / \mathrm{O}_{2}$ cell. The type B electrocatalyst is evaluated at three different potentials ( 0.84 , $0.70,0.40 \mathrm{~V}$ RHE) in a single $\mathrm{H}_{2} / \mathrm{O}_{2}$ cell at $80^{\circ} \mathrm{C}$. [37] 

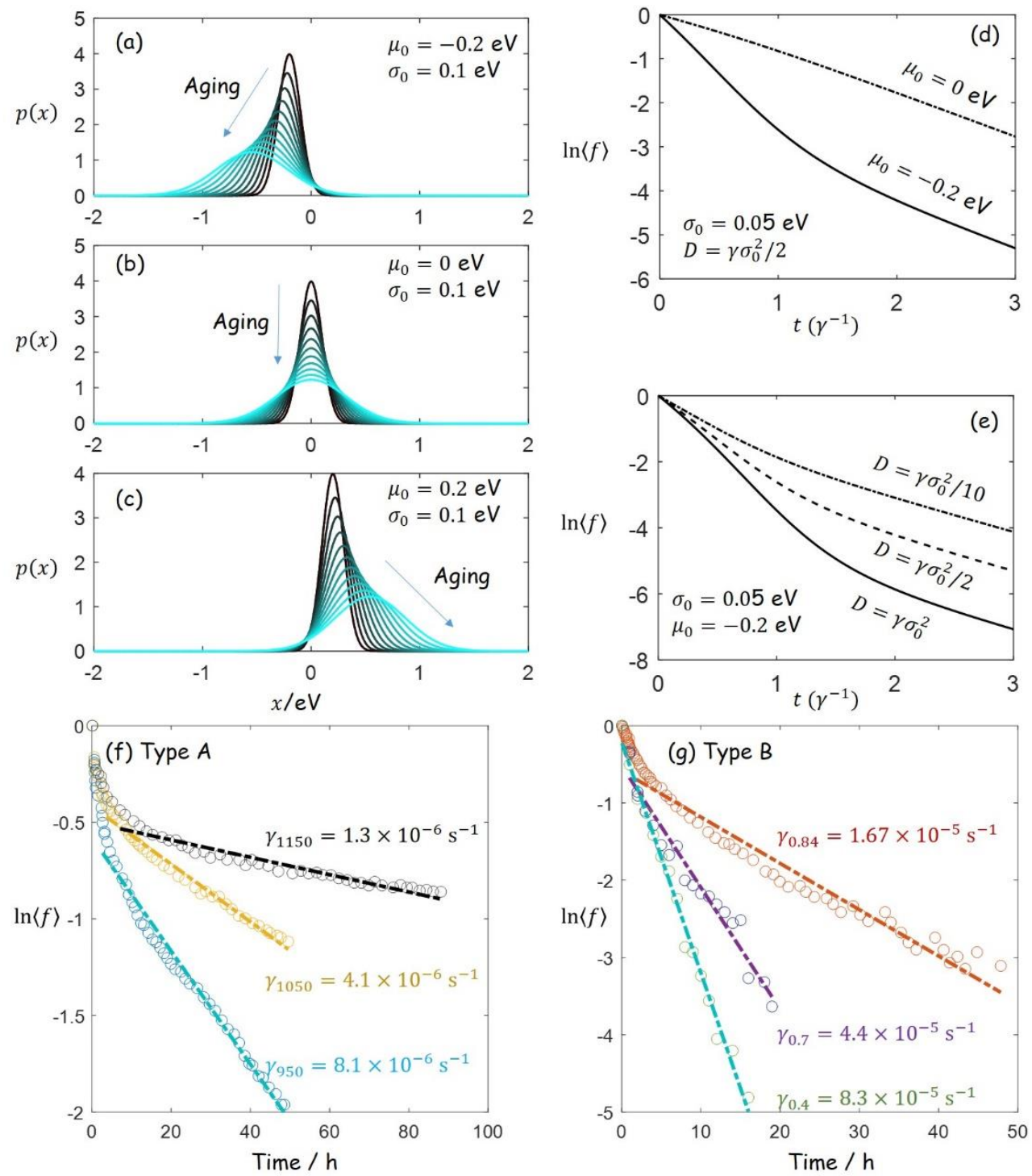

Figure 4. (a)-(c) Evolution of the probability distribution function from different initial conditions. The diffusion coefficient is $D=0.005\left(\mathrm{~m} \mathrm{eV}^{-2} \mathrm{~s}^{-1}\right)$, the time range is $\left(0, \gamma^{-1}\right)$, and the time step is $(10 \gamma)^{-1}$. Evolution of $\langle f\rangle$ normalized to its initial value for (d) different initial mean values $\mu_{0}$ and (e) different diffusion coefficients $D$ with other conditions specified. (f) and (g) Fitting degradation behavior of platinum group metal-free (PGM-free) oxygen reduction electrocatalysts using the developed theory. Dashed lines represent model simulation, and symbols represent experimental data which have been normalized with respect to their initial values. (f) shows the results for NC-CITeTMPP electrocatalyst (type A) prepared by Shao et al.[34] Three pyrolysis temperatures $\left(950,1050,1150{ }^{\circ} \mathrm{C}\right)$ are compared. The degradation tests were conducted using a single $\mathrm{H}_{2} / \mathrm{O}_{2}$ cell at $0.6 \mathrm{~V}_{\text {RHE}}$. (g) shows the results for CM-PANI-Fe-C( $\mathrm{Zn}$ ) electrocatalyst (type B) prepared by Yin and Zelenay.[37] The degradation tests were conducted using a single $\mathrm{H}_{2} / \mathrm{O}_{2}$ cell at $80^{\circ} \mathrm{C}$ at three different potentials $\left(0.84,0.70,0.40 \mathrm{~V}_{\mathrm{RHE}}\right)$.

A two-stage decay pattern, consisting of an initial fast stage and a successive slow stage, is found for the two electrocatalysts, falling into the regime of $\left|\mu_{0}\right|>\beta \sigma_{0}^{2}$ in the present theory. $\mu_{0}$ and $\sigma_{0}$ of these two electrocatalysts are not reported in the literature. The notorious ill-defined nature and variety of active sites prevent us from a reliable 
determination of $\mu_{0}$ and $\sigma_{0}$ using electronic structure calculations. Thus, focus is put on the second stage, which follows a relation of $\ln \langle f\rangle \propto-\gamma t$ according to the present theory. From the second stage, values for $\gamma$ are obtained unambiguously, and listed beside the corresponding curve in Figure 4. It is found that, $\gamma$ is lower, indicating retarded deactivation of active sites and slower degradation, when the pyrolysis temperature is higher and when the overpotential is lower; both trends are intuitively reasonable, lending credence to the presented simple theory.

\section{Conclusion}

We developed a simple theory with exact analytical solutions to describe statistical properties of electrocatalytic activity and durability. Our theory demonstrates that the overall activity of a nonhomogeneous electrocatalyst is strongly dependent on the inhomogeneity and standard deviation of the active sites. More precisely, the highest overall activity is obtained when the standard deviation is commensurate with the mean value of the binding energy, thus nonhomogeneity is not always detrimental. The site-site variation of activity is derived, which can be used as an analytical tool for electrocatalytic activity mapping methods. Degradation patterns are analytically related to the evolution kinetics of the PDF via the Fokker-Planck theory. Exponential decay of the overall activity is predicted by our theory and confirmed by experimental data of ORR catalysts taken from the literature. Considering that statistical considerations are urgently needed for electrocatalyst design, our theory, the first one of its sort, can be an important theoretical tool to characterize and further to design the distribution at the active-site level.

\section{Acknowledgement}

This work is financially supported by the Alexander von Humboldt Foundation, and National Natural Science Foundation of China under the grant number of 21802170. The author is grateful to Dr. Kai Exner for insightful discussions.

\section{References and Notes}

[1] J. K. Nørskov, T. Bligaard, B. Hvolbæk, F. Abild-Pedersen, I. Chorkendorff, and C. H. Christensen, Chemical Society Reviews 37, 2163 (2008).

[2] E. Herrero and J. M. Feliu, Current Opinion in Electrochemistry 9, 145 (2018).

[3] M. T. M. Koper, Nanoscale 3, 2054 (2011).

[4] N. M. Marković and P. N. Ross, Surface Science Reports 45, 117 (2002).

[5] A. S. Bandarenka and M. T. M. Koper, Journal of Catalysis 308, 11 (2013).

[6] V. Čolić and A. S. Bandarenka, ACS Catalysis 6, 5378 (2016).

[7] S. Kolagatla, P. Subramanian, and A. Schechter, Applied Catalysis B: Environmental 256, 117843 (2019).

[8] S. Kolagatla, P. Subramanian, and A. Schechter, ChemSusChem 12, 2708 (2019).

[9] A. J. Wain, Electrochimica Acta 92, 383 (2013).

[10] Y.-C. Weng and C.-T. Hsieh, Electrochimica Acta 56, 1932 (2011).

[11] C. M. Sánchez-Sánchez, F. J. Vidal-Iglesias, J. Solla-Gullón, V. Montiel, A. Aldaz, J. M. Feliu, and E. Herrero, Electrochimica Acta 55, 8252 (2010).

[12] Y. Wang, E. Gordon, and H. Ren, Analytical Chemistry 92, 2859 (2020).

[13] F. Calle-Vallejo, M. D. Pohl, D. Reinisch, D. Loffreda, P. Sautet, and A. S. Bandarenka, Chemical Science 8, 2283 (2017).

[14] J. K. Nørskov et al., Journal of Catalysis 209, 275 (2002). 
[15] B. Hammer and J. K. Norskov, Nature 376, 238 (1995).

[16] A. J. Appleby, Catalysis Reviews 4, 221 (1971).

[17] S. Trasatti, Journal of Electroanalytical Chemistry and Interfacial Electrochemistry 39, 163 (1972).

[18] J. Rossmeisl, Z. W. Qu, H. Zhu, G. J. Kroes, and J. K. Nørskov, Journal of Electroanalytical Chemistry 607, 83 (2007).

[19] F. Abild-Pedersen, J. Greeley, F. Studt, J. Rossmeisl, T. R. Munter, P. G. Moses, E. Skúlason, T. Bligaard, and J. K. Nørskov, Physical Review Letters 99, 016105 (2007).

[20] K. S. Exner, ACS Catalysis 9, 5320 (2019).

[21] T. Bligaard, J. K. Nørskov, S. Dahl, J. Matthiesen, C. H. Christensen, and J. Sehested, Journal of Catalysis 224, 206 (2004).

[22] J. K. Nørskov, T. Bligaard, J. Rossmeisl, and C. H. Christensen, Nature Chemistry 1, 37 (2009).

[23] I. E. L. Stephens, A. S. Bondarenko, U. Grønbjerg, J. Rossmeisl, and I. Chorkendorff, Energy \& Environmental Science 5, 6744 (2012).

[24] S. G. Rinaldo, J. Stumper, and M. Eikerling, The Journal of Physical Chemistry C 114, 5773 (2010).

[25] S. G. Rinaldo, P. Urchaga, J. Hu, W. Lee, J. Stumper, C. Rice, and M. Eikerling, Physical Chemistry Chemical Physics 16, 26876 (2014).

[26] S. G. Rinaldo, W. Lee, J. r. Stumper, and M. Eikerling, Electrochemical and Solid-State Letters 14, B47 (2011).

[27] Y. Zhang, J. Zhang, and J. Huang, The Journal of Physical Chemistry Letters 10, 7037 (2019).

[28] K. S. Exner, ChemCatChem 11, 3234 (2019).

[29] J. Chen, Y. Chen, P. Li, Z. Wen, and S. Chen, ACS Catalysis 8, 10590 (2018).

[30] H. Ooka and R. Nakamura, The Journal of Physical Chemistry Letters 10, 6706 (2019).

[31] U. Martinez, S. Komini Babu, E. F. Holby, and P. Zelenay, Current Opinion in Electrochemistry 9, 224 (2018).

[32] T. Asset and P. Atanassov, Joule 4, 33 (2020).

[33] L. Du, V. Prabhakaran, X. Xie, S. Park, Y. Wang, and Y. Shao, Advanced Materials n/a, 1908232 (2020).

[34] M. Shao, Q. Chang, J.-P. Dodelet, and R. Chenitz, Chemical Reviews 116, 3594 (2016).

[35] A. Nitzan, Chemical dynamics in condensed phases: relaxation, transfer and reactions in condensed molecular systems (Oxford university press, 2006).

[36] H. Chen, Z. Song, X. Zhao, T. Zhang, P. Pei, and C. Liang, Applied Energy 224, 289 (2018).

[37] X. Yin and P. Zelenay, ECS Transactions 85, 1239 (2018). 